\title{
Self-Assembled Hierarchical Nanostructures for High-Efficiency Porous Photonic Crystals
}

\author{
Luca Passoni, ${ }^{t, \neq, \S}$ Luigino Criante, ${ }^{t, \S}$ Francesco Fumagalli, ${ }^{\dagger}$ Francesco Scotognella, ${ }^{t, \neq}$ Guglielmo Lanzani, ${ }^{t, \neq}$ \\ and Fabio Di Fonzo*,t \\ ${ }^{\dagger}$ Center for Nano Science and Technology@PoliMi, Istituto Italiano di Tecnologia, Via Giovanni Pascoli, 70/3, 20133 Milano, Italy and ${ }^{\ddagger}$ Dipartimento di Fisica, \\ Politecnico di Milano, Piazza Leonardo da Vinci 32, 20133 Milano, Italy. ${ }^{\S}$ L. Passoni and L. Criante equally contributed to this work.
}

M other-of-pearls, opals, and butterfly wings are natural examples of photonic structures showing structural color: interference between light waves reflected by periodic or random subwavelength domains determines a range of forbidden frequencies in transmission, known as photonic band gap (PBG). When domains are periodic, these materials are named photonic crystals. $^{1-7}$ Mimicking the natural world, several photonic structures with domain periodicities in one, ${ }^{8,9}$ two, ${ }^{10}$ and three dimensions $s^{7,11,12}$ have been realized. One-dimensional photonic crystals, or Bragg stacks, can be fabricated by several techniques (e.g., electrochemical etching, spin coating, and various physical vapor deposition (PVD) techniques). ${ }^{11,13}$ Refractive index contrast is usually obtained varying the chemical composition of the layers or by tuning the layers' porosity. An open and fully porous architecture with high surface area would represent an important step forward in the exploitation of photonic crystals. Porous photonic crystals couple selective mass transport of gaseous and liquid analytes through the mesostructures with an engineered photonic band structure. This can enable the realization of stimuli-responsive and tunable optical devices with a broad range of potential applications, such as filtering, sensing, electrooptical switches, light management in sensitized solar cells, and photocatalysis. ${ }^{14,15}$ While the physics behind porous one-dimensional photonic crystals (p1DPCs) has been intensively investigated and it is now well understood, the fabrication technologies still lag behind, suffering a number of limitations, namely, material choice, fine optical and structural quality, control and repeatability, scalability, and flexibility in device integration, most notably in the CMOS platform.

Extensive reviews can be found in the literature describing fabrication methods
* Address correspondence to Fabio.difonzo@iit.it.

Received for review July 8, 2014 and accepted November 21, 2014. 
for 1D porous photonic crystals as well as for higher dimensionality structures (i.e., 2D, opals, and other 3D photonic crystals). ${ }^{7,15,16}$

The historical top-down approach to p1DPC realization is electrochemical etching of conductive wafers (e.g., doped-Si, Al). ${ }^{17-19}$ In this scheme, modulation of the anodic current through a substrate immersed in an acidic solution allows pores to be etched with tunable size from nanometers to micrometers. ${ }^{19-21}$ Although this wet technique was perfected in the mid-1990s, it is limited to certain conductive materials, requires a complex and multistep fabrication, and generally yields layers with low refractive index (RI) contrast. In order to overcome this bottleneck, bottom-up assembly methods have been proposed by several authors with the aim of demonstrating many of the countless promises made by theory. The use of colloidal nanoparticle (NP) dispersions $\left(\mathrm{SiO}_{2}, \mathrm{TiO}_{2}, \mathrm{ZnO}\right.$, and $\mathrm{SnO})^{11,14,22,23}$ as p1DPC building blocks is probably the easiest and most widely used self-assembly approach. Careful selection of the NP material and size distribution allows fabrication of multilayers with high photonic band gap efficiency and thicknesses raging from micrometers (using two different materials) to millimeters (direct and inverse opal). Most of these structures have been fabricated by spin-coating processes. ${ }^{24-26}$ More recently, gas-phase fabrication of p1DPCs was demonstrated via glancing angle deposition (GLAD), ${ }^{27,28}$ a PVD technique that allows engineering of different film architectures by exploiting ballistic atomic shadowing of vapor atoms at highly oblique deposition angles. Precise control over individual layer RI and thickness and access to different materials are main advantages of GLAD. Nevertheless the choice of materials is somewhat limited to those that can be evaporated by an incident electron beam, while the complex mechanical manipulation of the substrate hinders large-area realizations. ${ }^{29}$ Although many interesting improvements have recently been obtained of the above-reported techniques, many requirements have not yet been simultaneously satisfied: control over optical quality, high surface area packing density, wide substrate and platform compatibility.

In this article, we report an alternative fabrication method based on the bottom-up self-assembly of elementary building blocks (i.e., atoms, molecules, clusters) into high surface area photonic hierarchical nanostructures at room temperature. In this study the process is demonstrated by means of a pulsed laser deposition (PLD) apparatus. Nevertheless, we believe this process is not strictly limited to PLD, but it can be achieved anytime that precise control over directionality and energy of an inseminated supersonic jet impinging on a substrate is obtained. Studies are under way in order to prove this statement. In a similar fashion to the beetle cuticle, ${ }^{30}$ the hierarchical nanostructures are engineered in order to achieve a periodic refractive index modulation in the direction normal to the substrate. The naturally occurring array of these quasi-1D nanostructures exhibits a photonic stop band while retaining the hierarchical nature and high surface area of its constituents. ${ }^{31,32}$ Moreover, the spacing in between single nanostructures forms a vertically oriented mesoporosity, easily accessible by gas and liquids. ${ }^{33,34}$ We named these ensembles hierarchical one-dimensional photonic crystals (h1DPCs) and demonstrated their potential by fabricating h1DPCs achieving $75 \%$ reflection efficiency with just four repeating bilayers (for a total thickness comparable to the Bragg peak wavelength of about $\sim 460 \mathrm{~nm}$ ). In accordance with the saturating behavior of the reflectance efficiency as a function of thickness, eight bilayers are necessary to reach a value close to unity. All these characteristics together with the facile realization of patterned photonic arrays on the length scale of few micrometers open interesting perspectives for applications in the fields of biochemical sensing, ${ }^{35}$ solar cells, displays, photoelectrochemical water splitting, and photocatalysis. Moreover, as PLD is a single step, low thermal budget technique, it is compatible with a wide variety of substrates, including plastic or biological materials. ${ }^{36,37}$

\section{RESULTS AND DISCUSSION}

In PLD (apparatus depicted in Figure SI 1) a pulsed excimer laser ( $\mathrm{KrF} \lambda=248$ ) is focused on a solid target material, generating a supersonic plasma plume expanding in a vacuum chamber where a background gas is set to a specific pressure. The interaction between the gas and the plume controls cluster nucleation, kinetic energy, and directionality. This in turn controls the morphological characteristics of the deposited film. As an example, the lower the pressure, the lower the cluster mean size and their collisional scattering by the background gas and the higher their kinetic energy, thus yielding dense films. Conversely, high pressures favor the growth of hierarchical nanostructures and hence of a porous film. As demonstrated in previous publications, ${ }^{31,32,38-40}$ chemical and morphological parameters of complex hierarchical nanostructures can be finely controlled. Moreover, high specific surface areas, as measured by the Brunauer-Emmett-Teller (BET) method, in the range of $100-300 \mathrm{~m}^{2} / \mathrm{g}$ and high pore volume density are obtained for each single layer (see Supporting Information and Figure SI 2). In this study, a periodic dielectric function contrast is achieved modulating the density of the hierarchical quasi-1D nanostructures during their growth. A schematic of layer formation together with a high-magnification SEM image of the array of hierarchical nanostructures with clear evidence of density modulation is shown in Figure 1.

An important aspect to take into account for a precise control of the RI modulation is the influence 

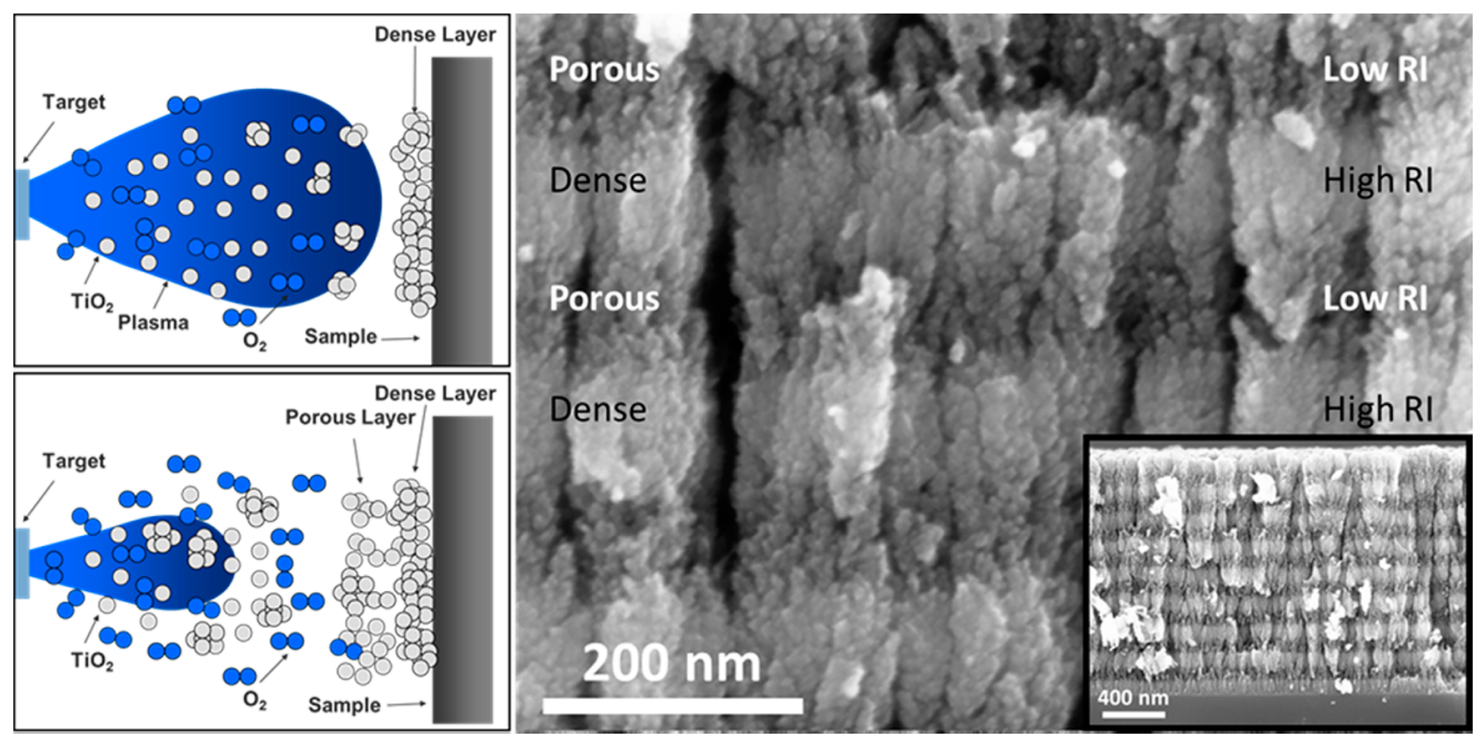

Figure 1. (Left) Schematic of layer formation by pulsed laser deposition. (Top) In the case of low pressure a dense film is formed due to high-energy species present in the plasma. (Bottom) If the pressure is increased, more porous material is deposited. (Right) High-resolution SEM showing details of the periodic layer stack constituent of the (inset) complete photonic crystals.

of each layer on the following during growth (see details in the Supporting Information and Figure SI

3). This effect makes the pressure-density relationship for layers grown individually on a substrate imprecise in this context. In order to avoid a tedious trial and error experimental procedure in a multiparametric space (i.e., porosity, refractive index, and thickness of each layer), we used the well-established transfer matrix method (TMM) ${ }^{41}$ to simulate the transmission spectra of the photonic crystals in order to extract the optical parameters of the individual layers and, indirectly, their effective density and porosity. ${ }^{42}$ Hence, a library of couples of layers grown at different pressures (P1, P2) was first established and then used to precisely design even complex h1DPCs in a repeatable way. The method can be readily extended to the case of a multimaterial system. As an example of successful implementation of this procedure, we show in Figure 2 three different $\mathrm{TiO}_{2}$ h1DPCs fabricated keeping the pressure (P1) of the first layer (L1) constant (high n, lower porosity) and varying pressure (P2) of the second layer (L2) (low $n$, higher porosity). The simulations (red curves in Figure 2 right) are in good agreement with the observed transmission spectra (black dots, Figure 2 right). A quality index testifying the correspondence of the simulations with reality is the precise matching between experimental and calculated lateral fringes. The notch depth of the photonic stop bands highlighted in the spectral behavior (Figure 2 right) is the direct expression of the real diffraction efficiency of the photonics crystal. As the deposition pressure of layer L1 is kept constant, also its porosity (and thus refractive index) does not change substantially across different photonic crystals. On the other hand, the porosity of layer L2 has been monotonically increased by increasing the deposition pressure, thus decreasing its refractive index. Taking into account the substrate (glass-FTO) intrinsic losses (dashed gray line), the reflection performance of sample c approaches $100 \%$ with a device thickness of only $\sim 1.5 \mu \mathrm{m}$. The values of refractive index and porosity extrapolated from the fit performed on the experimental data are reported in Figure 3. The refractive index modulation reaches a value close to unity in the most extreme case $(5,20)$ shown in Figure SI 4 of the Supporting Information (refractive index contrasts $\Delta n_{(\mathrm{L} 1, \mathrm{~L} 2)}$ of $\Delta n_{(5,7)} \approx 0.4$, $\Delta n_{(5,10)} \approx 0.6, \Delta n_{(5,15)} \approx 0.7$, and $\left.\Delta n_{(5,20)} \approx 0.9\right)$. Indeed, for an equal number of bilayers, as the difference between two refractive indices, $\Delta n_{(\mathrm{L} 1, \mathrm{~L} 2)}$, increases, diffraction becomes more efficient and the reflection peak expands its bandwidth (fwhm) in good agreement with photonic crystal theory. It is worth noticing that the coupling effect between different layers is quite uniform up to a P2 of $15 \mathrm{~Pa}$, as testified by the relatively constant porosity and $\mathrm{RI}$ of $\mathrm{L} 1$, while it becomes more severe in the $(5,20)$ case.

It is noteworthy that by maximizing the refractive index contrast with only four bilayers $(\sim 460 \mathrm{~nm}$ ), a reflection efficiency of over $75 \%$ can be achieved (Figure 4a (SEM image) and Figure 4b (transmission spectrum)). By means of comparison, other monomaterial p1DPCs, fabricated by GLAD or anodization techniques, have shown reflection efficiencies close to $80 \%$ with thicknesses ranging from nine to 30 bilayers $(1.7$ and $8.2 \mu \mathrm{m})$, respectively. ${ }^{27,43}$ In general the PBG spectral position and width are arbitrarily tunable. As an example, an h1DPC in the telecom spectral range was realized (see Supporting Information Figure SI 5). A broadband (400-800 nm) h1DPC with reflectivity close to unity was designed superimposing four 

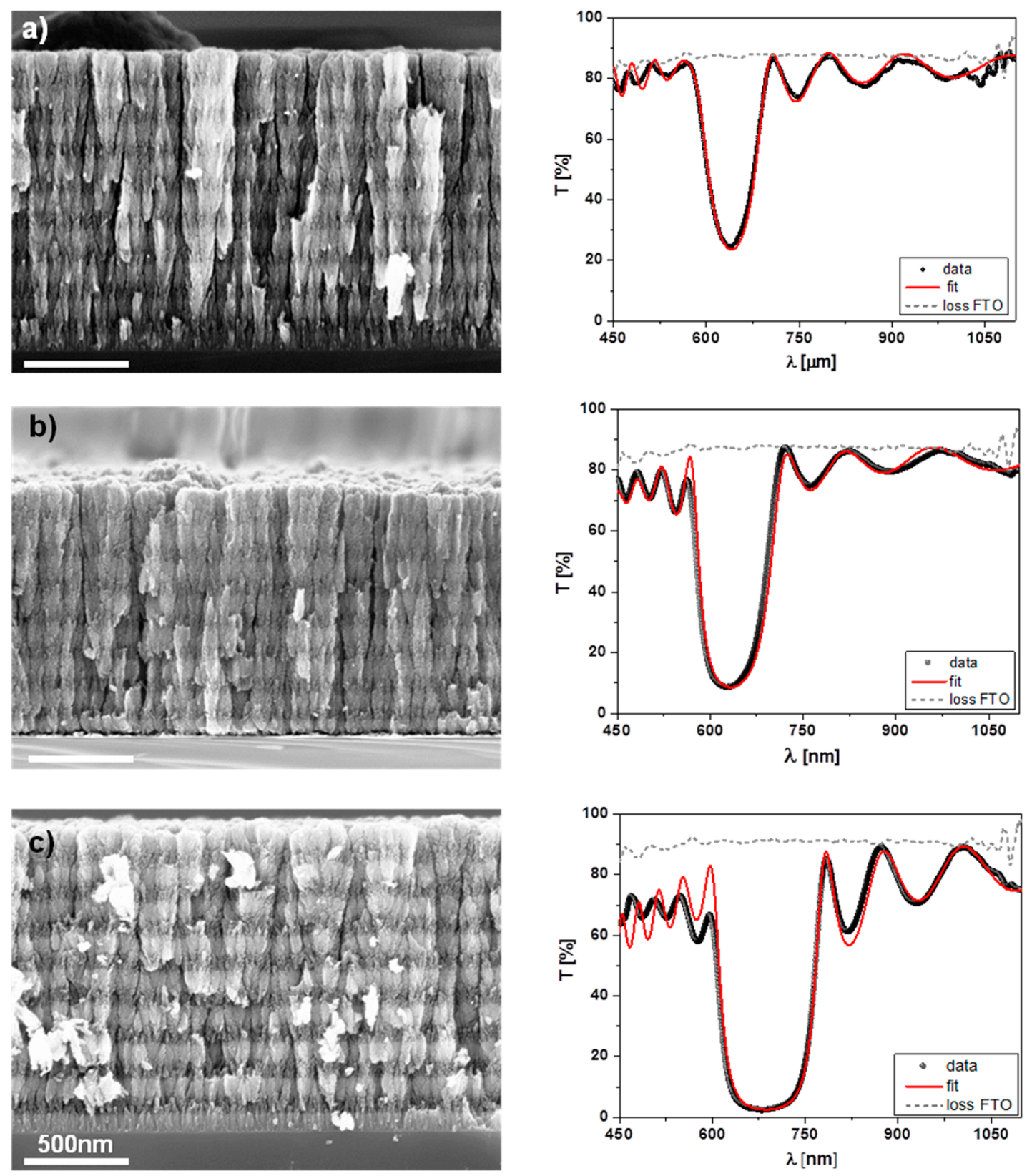

Figure 2. Scanning electron microscope images and transmission spectra (data and best fit) of three hierarchical photonic crystals with increasing density and refractive index contrast of the base unit forming the photonic crystal. The pressure value are $(5,7),(5,10)$, and $(5,15)$ for samples $a$, b, and c, respectively.

$\mathrm{TiO}_{2}$ photonic crystals with complementary PBG (see Figure $4 \mathrm{c}$ and $\mathrm{d}$ ).

Control over the optical characteristics of the h1DPC upon thermal treatment is of paramount importance for many applications in which the device fabrication process requires high-temperature steps, in order to achieve good crystalline quality and efficient electronic transport (e.g., dye-sensitized and perovskite solar cells, water splitting, ${ }^{44}$ optoelectronic switches, and sensors). To simulate this, we annealed a PLD-fabricated h1DPC at $T=500^{\circ} \mathrm{C}$. The optical properties were seen to be preserved with only a minor blue shift in photonic band gap energy due to a structural shrinking of the layer caused by local densification/crystallization processes (Supporting Information Figure SI 6). Moreover, the high roughness factor of the photonic crystal (i.e., available surface area over the geometrical area of the sample, RF) was also retained. An RF value of $145 \mu \mathrm{m}^{-1}$ was found for as-deposited samples through chromatographic analysis of the desorbed dye. For annealed samples the roughness factor decreased to $\mathrm{RF}=100 \mu \mathrm{m}^{-1}$ (more details in the Supporting Information), typical of state-of-the-art dye-sensitized solar cell photoanodes.

A further advantage of our process is the facile fabrication of patterned porous photonic crystals by 
means of a simple shadow mask (Figure 5a). Here the photonic crystal was grown off-axis with respect to the deposition source in such a way as to grade the multilayer along the letters, giving rise to a blue shift (from top to bottom) of the photonic band gap (Figure 5b).

The potential to make patterns was further exploited to realize a pixel structure of porous photonic crystals

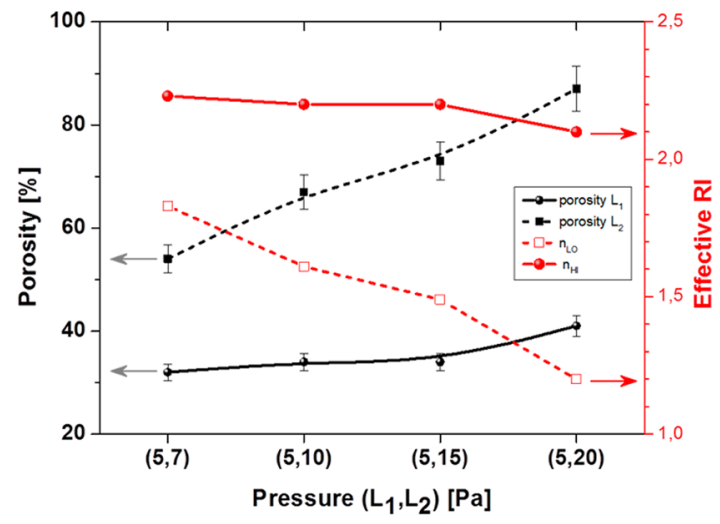

Figure 3. Porosity and effective refractive index (RI) of the two photonic crystal layers. $L_{1}$ and $L_{2}$ are the low and high porosity layer having respectively refractive index $n_{\mathrm{HI}}$ and $n_{\mathrm{LO}}$. in which each pixel has a size of approximately 10 by $10 \mu \mathrm{m}$ (Figure 5c). Such pixel dimensions fits well the size required in display technologies, and these could be applied for sensing purposes. On the other hand being intrinsically a room-temperature technique, PLD allows the fabrication of h1DPCs on a flexible plastic substrate (Figure $4 d$ ) of interest for organic electronics.

The large oriented porosity of the h1DPC can be exploited for measuring the refractive index of fluid substances, paving the way for novel optoelectronic devices and sensors. ${ }^{45}$ As an example, acetone (with a refractive index ranging from 1.354 and 1.364 in the spectral region considered) was infiltrated into the h1DPC. In Figure 6 the red shift due to acetone infiltration is shown together with the evaporation process demonstrating the restoration of the original PBG. In Figure $6 \mathrm{~b}$ we show the infiltration of E7 liquid crystals, which have an isotropic refractive index of about 1.575 in the visible. Infiltration in this case also gives rise to a red shift of the photonic band gap. These examples demonstrate the possibilities for building nanostructured composite materials for diverse optical applications, such as lasing, emitting diodes, sensors, and sensitized photovoltaic cells.
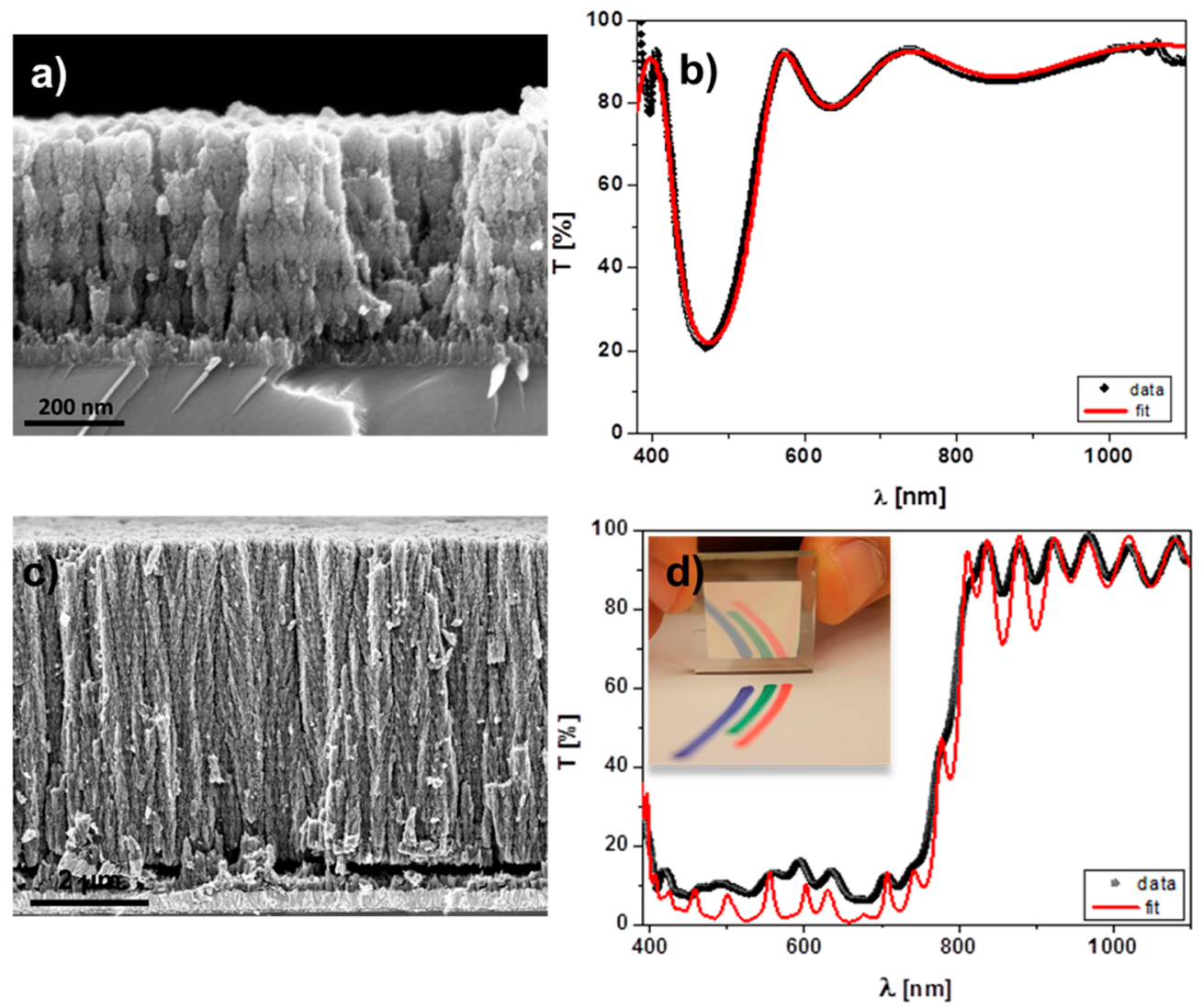

Figure 4. (a) Scanning electron microscope image and (b) transmission spectra (data and best fit) of a four-bilayer, $\sim 460 \mathrm{~nm}$ thick $\mathrm{TiO}_{2}$ hierarchical photonic crystal achieving over $75 \%$ reflectivity. (c) Scanning electron microscope image and (d) transmission spectra (data and best fit) of $\mathrm{TiO}_{2}$ porous photonic crystals with reflection band $(R \approx 1)$ over the whole visible spectrum obtained by superposition of four different photonic crystals with different pitches. In the inset the picture shows its mirror-like appearance. 

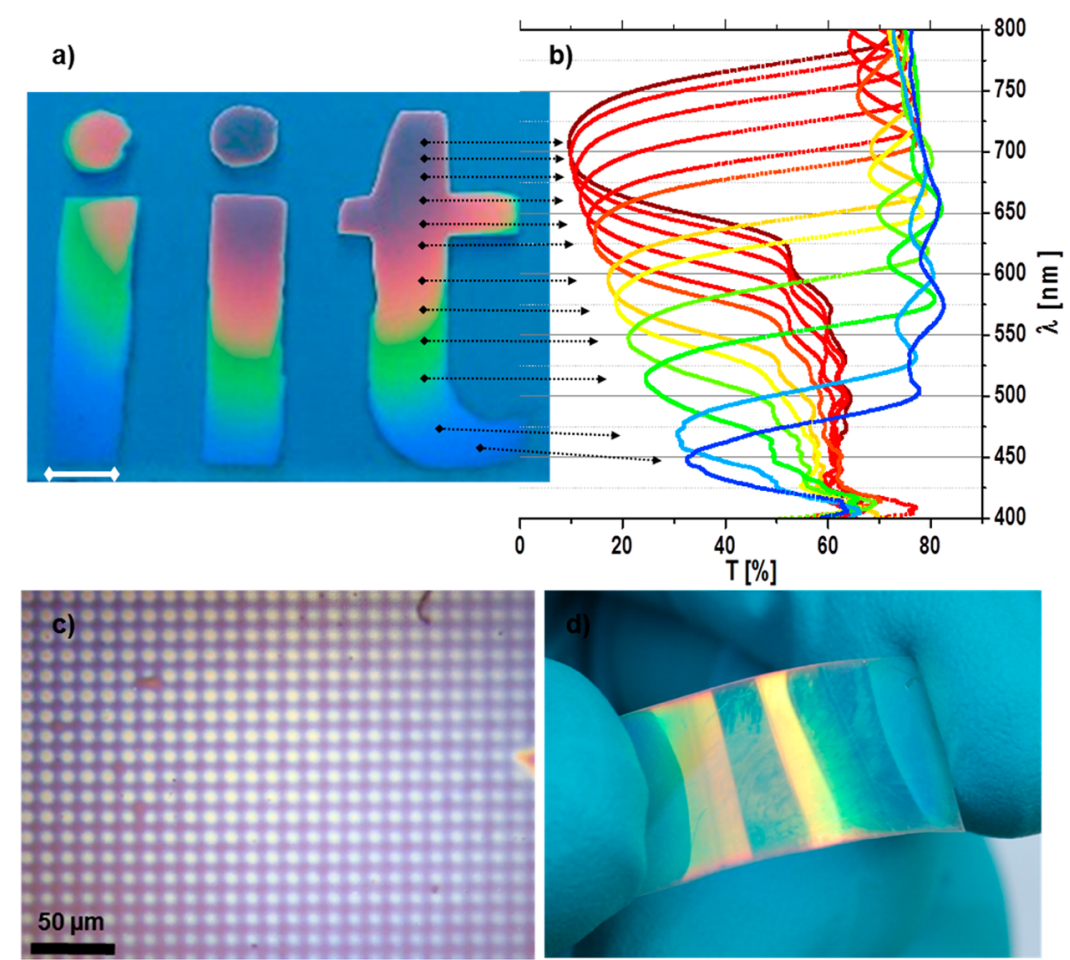

Figure 5. (a) Patterned photonic crystals, fabricated with a thickness gradient of the layers, by using a mask. Scale bar: $1 \mathrm{~cm}$. (b) Transmission spectra of the photonic crystal along one of the letters, from top to bottom; a shift of the photonic band gap spectral position of a few hundred of nanometers is shown. (c) Pixel structure of a hierarchical photonic crystal. (d) h1DPC deposited on a flexible plastic substrate.
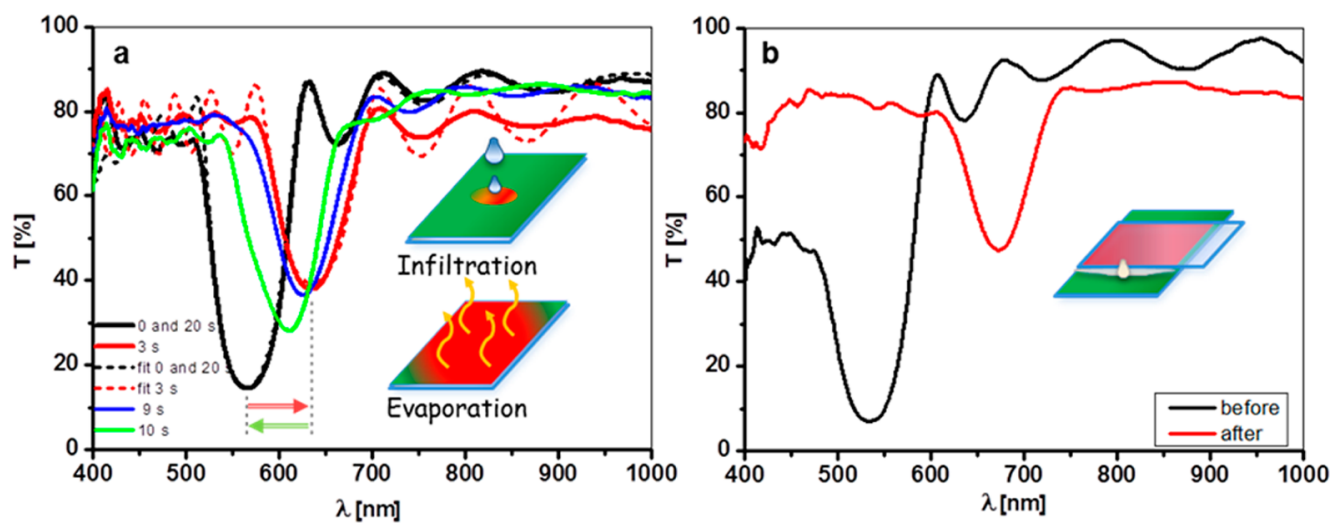

Figure 6. Measurements of transmission spectra of all $\mathrm{TiO}_{2}$ hierarchical one-dimensional photonic crystals (a) during the infiltration process with acetone (dashed lines denote transfer matrix simulations) and transmission measurements of its subsequent evaporation; (b) effect on the transmission spectra of the infiltration with liquid crystals.

\section{CONCLUSIONS}

Arrays of hierarchical photonic nanostructures selfassembled from the gas phase have been successfully grown to form high-efficiency one-dimensional porous photonic crystals by PLD. This new tool for nanophotonic fabrication permits (a) the fabrication of photonic crystals with high efficiency/thickness ratio; (b) the realization of photonic sequences with fine control of the optical thickness of each layer; (c) the creation of patterned h1DPC structures with macro- and microdimensions using simple shadow masks without recourse to lithographic processes; (d) the fabrication of ultrabroadband dielectric mirrors. Our technology is robust with respect to thermal treatments and is potentially compatible with silicon and plastic electronics (Figure SI 6 and Figure 5d, respectively), possibly paving the way to advanced integrated photonic circuits. The photonic crystals we obtained comprise layered hierarchical quasi 1D nanostructures with controlled porosity that can be used as sensor arrays ${ }^{46}$ or as a handle for optical modulation. With this fabrication strategy we envisage large-scale, low-cost production of photonic devices on rigid and flexible substrates with fine-tuning of spectral range, porosity, and material composition for a myriad of applications. 
Impacted technological areas span from filtering and light harvesting to active materials such as lasers,

\section{METHODS}

PLD Deposition. In Supporting Information Figure S1 the experimental setup for pulse laser deposition is shown. This technique comprises a pulsed excimer laser ( $\mathrm{KrF} \lambda=248 \mathrm{~nm})$ focused through an optical path on a target of a desired material $\left(\mathrm{TiO}_{2}\right)$ that is vaporized. The ablated material, in the form of atoms and molecules, recondenses on the substrate (S) placed perpendicularly in front of the target. The deposition takes place in a vacuum chamber where a background gas is set at a specific pressure by mass flow meters. Laser fluence is set at $\sim 2 \mathrm{~J} / \mathrm{cm}^{2}$ with an energy per pulse of $400 \mathrm{~mJ}$ at a repetition rate of $20 \mathrm{~Hz}$. Sample to target distance is kept equal to $5 \mathrm{~cm}$ while the sample holder is rotated at $10 \mathrm{rpm}$ (sample tangential velocity $2 \mathrm{~cm} / \mathrm{s}$ ) in order to deposit a larger area.

Morphological Characterization. Samples have been analyzed by an Alpha Step profilometer and scanning electron microscope (SEM). SEM images are taken with a Zeiss Supra 40 field emission scanning electron microscope. Roughness factor per unit thickness (i.e., the ratio between effective and geometric surface area per micrometer of deposited film) was measured by the dye loading/unloading method. The colorimetric experiment was done on the desorbed dye (N719).

Optical Characterization. Optical characterization has been performed by using a tungsten lamp and concave grating spectrometer from Stellarnet (spectral resolution $1.5 \mathrm{~nm}$ ). For the spectral response in the transfer matrix method simulations, a Sellmeier equation for the refractive index of titanium dioxide has been taken into account (refractiveindex.info).

Conflict of Interest: The authors declare no competing financial interest.

Supporting Information Available: PLD setup scheme, BET measurements, UV/vis measurements after thermal treatment, roughness factor measurement methods, h1DPCs in telecom spectral range. This material is available free of charge via the Internet at http://pubs.acs.org.

Acknowledgment. G.L. and F.D.F. acknowledge funding from the FP7-Energy-2012, Collaborative Project, "Photogenerated Hydrogen by Organic Catalytic Systems (PHOCS)", Grant No. 309223. F.F. and F.D.F. acknowledge funding from "Photovoltaic Building Integrated Glass Tiles for Innovative Architectural Application" (TIFAIN), MIUR-Regione Lombardia ID number 30221157. The authors wish to thank L. Lietti (Department of Energy, Politecnico di Milano) for BET measurements and Marcelo J. P. Alcocer for revising the manuscript. F.D.F., F.S., and G.L. conceived the experiment. L.C. designed the devices. L.P., L.C., and F.F. carried out the experiment. L.P. and L.C. analyzed the data.

\section{REFERENCES AND NOTES}

1. Yablonovitch, E. Inhibited Spontaneous Emission in SolidState Physics and Electronics. Phys. Rev. Lett. 1987, 58, 2059-2062.

2. Joannopoulos, J. D.; Villeneuve, P. R.; Fan, S. Photonic Crystals: Putting a New Twist on Light. Nature 1997, 386, 143-149.

3. John, S. Strong Localization of Photons in Certain Disordered Dielectric Superlattices. Phys. Rev. Lett. 1987, 58, 2486-2489.

4. Sakoda, K. Optical Properties of Photonic Crystals; Springer: Berlin, 2005.

5. Galisteo-López, J. F.; Ibisate, M.; Sapienza, R.; FroufePérez, L. S.; Blanco, A.; López, C. Self-Assembled Photonic Structures. Adv. Mater. 2011, 23, 30-69.

6. Lee, J.-H.; Singer, J. P.; Thomas, E. L. Micro/Nanostructured Mechanical Metamaterials. Adv. Mater. 2012, 24, 47824810 . sensitized solar cells, ${ }^{47}$ and color pixels for display technology. $^{48}$
7. López, C. Materials Aspects of Photonic Crystals. Adv. Mater. 2003, 15, 1679-1704.

8. Smirnov, J. R. C.; Calvo, M. E.; Míguez, H. Selective UV Reflecting Mirrors Based on Nanoparticle Multilayers. Adv. Funct. Mater. 2013, 23, 2805-2811.

9. Yang, Q.; Zhu, S.; Peng, W.; Yin, C.; Wang, W.; Gu, J.; Zhang, W.; Ma, J.; Deng, T.; Feng, C.; et al. Bioinspired Fabrication of Hierarchically Structured, pH-Tunable Photonic Crystals with Unique Transition. ACS Nano 2013, 7, 4911-4918.

10. Kim, M. H.; Im, S. H.; Park, O. O. Rapid Fabrication of Two- and Three-Dimensional Colloidal Crystal Films via Confined Convective Assembly. Adv. Funct. Mater. 2005, 15, 1329-1335.

11. Bonifacio, L. D.; Lotsch, B. V.; Puzzo, D. P.; Scotognella, F.; Ozin, G. A. Stacking the Nanochemistry Deck: Structural and Compositional Diversity in One-Dimensional Photonic Crystals. Adv. Mater. 2009, 21, 1641-1646.

12. Wijnhoven, J. E. G. J.; Vos, W. L. Preparation of Photonic Crystals Made of Air Spheres in Titania. Science 1998, 281, 802-804.

13. Valligatla, S.; Chiasera, A.; Varas, S.; Bazzanella, N.; Rao, D. N.; Righini, G. C.; Ferrari, M. High Quality Factor 1-D Er3+-Activated Dielectric Microcavity Fabricated by RF-Sputtering. Opt. Express 2012, 20, 21214-21222.

14. Colodrero, S.; Ocana, M.; Miguez, H. Nanoparticle-Based One-Dimensional Photonic Crystals. Langmuir 2008, 24, 4430-4434.

15. Colodrero, S.; Ocaña, M.; González-Elipe, A. R.; Míguez, H. Response of Nanoparticle-Based One-Dimensional Photonic Crystals to Ambient Vapor Pressure. Langmuir 2008, 24, 9135-9139.

16. von Freymann, G.; Kitaev, V.; Lotsch, B. V.; Ozin, G. A. Bottom-Up Assembly of Photonic Crystals. Chem. Soc. Rev. 2013, 42, 2528-2554.

17. Turner, D. R. Electropolishing Silicon in Hydrofluoric Acid Solutions. J. Electrochem. Soc. 1958, 105, 402-408.

18. Guo, D.-L.; Fan, L.-X.; Wang, F.-H.; Huang, S.-Y.; Zou, X.-W. Porous Anodic Aluminum Oxide Bragg Stacks as Chemical Sensors. J. Phys. Chem. C 2008, 112, 17952-17956.

19. Unagami, T. Formation Mechanism of Porous Silicon Layer by Anodization in HF Solution. J. Electrochem. Soc. 1980, $127,476-483$.

20. Vincent, G. Optical Properties of Porous Silicon Superlattices. Appl. Phys. Lett. 1994, 64, 2367-2369.

21. Frohnhoff, S.; Berger, M. G. Porous Silicon Superlattices. Adv. Mater. 1994, 6, 963-965.

22. Singer, K. D.; Kazmierczak, T.; Lott, J.; Song, H.; Wu, Y.; Andrews, J.; Baer, E.; Hiltner, A.; Weder, C. Melt-Processed All-Polymer Distributed Bragg Reflector Laser. Opt. Express 2008, 16, 10358-10363.

23. Puzzo, D. P.; Bonifacio, L. D.; Oreopoulos, J.; Yip, C. M.; Manners, l.; Ozin, G. A. Color from Colorless Nanomaterials: Bragg Reflectors Made of Nanoparticles. J. Mater. Chem. 2009, 19, 3500-3506.

24. Floch, H. G.; Priotton, J.-J.; Thomas, I. M. Optical Coatings Prepared from Colloidal Media. Thin Solid Films 1989, 175, 173-178.

25. Thomas, I. M. Single-Layer $\mathrm{TiO}_{2}$ and Multilayer $\mathrm{TiO}_{2}-\mathrm{SiO}_{2}$ Optical Coatings Prepared from Colloidal Suspensions. Appl. Opt. 1987, 26, 4688-4691.

26. Chiappini, A.; Armellini, C.; Chiasera, A.; Ferrari, M.; Fortes, L.; Clara Gonçalves, M.; Guider, R.; Jestin, Y.; Retoux, R.; Nunzi Conti, G.; et al. An Alternative Method to Obtain Direct Opal Photonic Crystal Structures. J. Non-Cryst. Solids 2009, 355, 1167-1170.

27. Steele, J. J.; van Popta, A. C.; Hawkeye, M. M.; Sit, J. C.; Brett, M. J. Nanostructured Gradient Index Optical Filter for High-Speed Humidity Sensing. Sens. Actuators, B 2006, 120, 213-219. 
28. Schubert, M. F.; Xi, J. Q.; Kim, J. K.; Schubert, E. F. Distributed Bragg Reflector Consisting of High- and Low-RefractiveIndex Thin Film Layers Made of the Same Material. Appl. Phys. Lett. 2007, 90, 141115-3.

29. Huang, Z.; Bai, F. Wafer-Scale, Three-Dimensional Helical Porous Thin Films Deposited at a Glancing Angle. Nanoscale 2014, 6, 9401-9409.

30. Parker, A. R.; Townley, H. E. Biomimetics of Photonic Nanostructures. Nat. Nanotechnol. 2007, 2, 347-353.

31. Passoni, L.; Ghods, F.; Docampo, P.; Abrusci, A.; Martí-Rujas, J.; Ghidelli, M.; Divitini, G.; Ducati, C.; Binda, M.; Guarnera; et al. Hyperbranched Quasi-1D Nanostructures for SolidState Dye-Sensitized Solar Cells. ACS Nano 2013, 7, 1002310031.

32. Sauvage, F.; Di Fonzo, F.; Li Bassi, A.; Casari, C. S.; Russo, V.; Divitini, G.; Ducati, C.; Bottani, C. E.; Comte, P.; Graetzel, M. Hierarchical $\mathrm{TiO}_{2}$ Photoanode for Dye-Sensitized Solar Cells. Nano Lett. 2010, 10, 2562-2567.

33. Chiappini, A.; Armellini, C.; Carpentiero, A.; Minati, L.; Righini, G. C.; Ferrari, M. Solvent Sensitive Polymer Composite Structures. Opt. Mater. 2013, 36, 130-134.

34. Di Fonzo, F.; Casari, C. S.; Russo, V.; Brunella, M. F.; Bassi, A. L.; Bottani, C. E. Hierarchically Organized Nanostructured $\mathrm{TiO}_{2}$ for Photocatalysis Applications. Nanotechnology 2009, 20.

35. Cunin, F.; Schmedake, T. A.; Link, J. R.; Li, Y. Y.; Koh, J.; Bhatia, S. N.; Sailor, M. J. Biomolecular Screening With Encoded Porous-Silicon Photonic Crystals. Nat. Mater. 2002, 1, 39-41.

36. Orlandi, M.; Caramori, S.; Ronconi, F.; Bignozzi, C. A.; Koura, Z. E.; Bazzanella, N.; Meda, L.; Miotello, A. Pulsed-Laser Deposition of Nanostructured Iron Oxide Catalysts for Efficient Water Oxidation. ACS Appl. Mater. Interfaces 2014, 6, 6186-6190.

37. Gower, M. C. Industrial Applications of Laser Micromachining. Opt. Express 2000, 7, 56-67.

38. Di Fonzo, F.; Casari, C. S.; Russo, V.; Brunella, M. F.; Li Bassi, A.; Bottani, C. E. Hierarchically Organized Nanostructured $\mathrm{TiO}_{2}$ for Photocatalysis Applications. Nanotechnology 2009, 20, 015604.

39. Dellasega, D.; Facibeni, A.; Di Fonzo, F.; Bogana, M.; Polissi, A.; Conti, C.; Ducati, C.; Casari, C. S.; Li Bassi, A.; Bottani, C. E. Nanostructured $\mathrm{Ag}_{4} \mathrm{O}_{4}$ films with Enhanced Antibacterial Activity. Nanotechnology 2008, 19, 475602.

40. Gonzalo, J.; Gómez San Román, R.; Perrière, J.; Afonso, C. N.; Pérez Casero, R. Pressure Effects During Pulsed-Laser Deposition of Barium Titanate Thin Films. Appl. Phys. A: Mater. Sci. Process. 1998, 66, 487-491.

41. Pendry, J. B. Symmetry and Transport of Waves in OneDimensional Disordered Systems. Adv. Phys. 1994, 43, 461-542.

42. Anaya, M.; Calvo, M. E.; Luque-Raigón, J. M.; Míguez, H. Resonant Photocurrent Generation in Dye-Sensitized Periodically Nanostructured Photoconductors by Optical Field Confinement Effects. J. Am. Chem. Soc. 2013, 135, 7803-7806.

43. Zheng, L.; Cheng, H.; Liang, F.; Shu, S.; Tsang, C. K.; Li, H.; Lee, S.-T.; Li, Y. Y. Porous TiO 2 Photonic Band Gap Materials by Anodization. J. Phys. Chem. C 2012, 116, 5509-5515.

44. Zhang, Z.; Yang, X.; Hedhili, M. N.; Ahmed, E.; Shi, L.; Wang, P. Microwave-Assisted Self-Doping of $\mathrm{TiO}_{2}$ Photonic Crystals for Efficient Photoelectrochemical Water Splitting. ACS Appl. Mater. Interfaces 2013, 6, 691-696.

45. Kobler, J.; Lotsch, B. V.; Ozin, G. A.; Bein, T. Vapor-Sensitive Bragg Mirrors and Optical Isotherms from Mesoporous Nanoparticle Suspensions. ACS Nano 2009, 3, 1669-1676.

46. Potyrailo, R. A.; Ghiradella, H.; Vertiatchikh, A.; Dovidenko, K.; Cournoyer, J. R.; Olson, E. Morpho Butterfly Wing Scales Demonstrate Highly Selective Vapour Response. Nat. Photonics 2007, 1, 123-128.

47. Varghese, L. T.; Xuan, Y.; Niu, B.; Fan, L.; Bermel, P.; Qi, M. Enhanced Photon Management of Thin-Film Silicon Solar Cells Using Inverse Opal Photonic Crystals with 3D Photonic Bandgaps. Adv. Opt. Mater. 2013, 1, 692-698.

48. Arsenault, A. C.; Puzzo, D. P.; Manners, I.; Ozin, G. A. Photonic-Crystal Full-Colour Displays. Nat. Photonics 2007, $1,468-472$. 